\title{
A PROSPECTIVE STUDY EXAMINING THE CHANGES IN FACTORS THAT AFFECT VISUAL ACUITY FOLLOWING TRABECULECTOMY
}

\author{
I. A. CUNLIFFE, R. B. DAPLING, J. WEST, S. LONGSTAFF \\ Sheffield
}

\begin{abstract}
SUMMARY
Many patients report subjective changes in vision following trabeculectomy. In a prospective study of 16 eyes, we have tried to determine the causes of these visual changes. Ninety-four per cent of eyes showed a change in uncorrected Snellen acuity 1 week following surgery. This change was due to a myopic shift in refraction secondary to changes in the anterior chamber depth. A $1 \mathrm{~mm}$ change in anterior chamber depth results in approximately a 2 dioptre change in refractive sphere. Visual acuity starts to return to pre-operative levels by the third post-operative week, from which point no eyes showed a deterioration in corrected acuity of more than one line of Snellen. The vertical corneal radius of curvature is reduced in the early post-operative period and there is a with-the-rule change in corneal astigmatism. This resolves over longer follow-up and the possible causes of this are discussed. No changes were noted in corneal thickness and there was no evidence of macular oedema over the follow-up period. Patients should be warned of these possible visual changes pre-operatively as many will have normal acuity prior to surgery.
\end{abstract}

Trabeculectomy was first described by Cairns ${ }^{1}$ in 1968 and later modified by Watson. ${ }^{2}$ Since then many studies have reported on its effectiveness in the control of intraocular pressure in various types of glaucoma. However, no study has assessed the early refractive changes following trabeculectomy and their effect on visual recovery in the early post-operative period. Many patients report changes in vision in the early post-operative period following trabeculectomy and it is important to warn them of these possible changes as operations are often performed on eyes with normal acuity. Watson ${ }^{3}$ has stated that many patients complain of an alteration in vision in the weeks following glaucoma surgery, but that it usually recovers fully within 6 weeks. The cause of this change was not clear, but it was

Correspondence to: Mr. I. A. Cunliffe, Department of Ophthalmology, Royal Hallamshire Hospital, Glossop Road, Sheffield S10 2JF UK. felt to be due to an alteration in the iris plane caused by the operation. In this study we have tried to determine the cause of these alterations in vision, to quantify any changes found, and to monitor these changes over a longer follow-up period.

\section{MATERIALS AND METHODS}

In a prospective study we examined 16 consecutive eyes of 16 patients undergoing trabeculectomy for chronic simple glaucoma. Where patients had both eyes operated on, only the first eye was included in the study. Entry criteria included a best corrected visual acuity of $6 / 12$ or better and no previous ocular surgery. Any patients who had narrow angle glaucoma, uveitis or significant maculopathy were excluded. Ethical committee approval was obtained for the study and patients gave their written informed consent. We measured several factors that may affect visual acuity pre-operatively and at 1 week, 3 weeks, 8 weeks and at a mean long-term follow-up of 10 months (minimum 6 months) following trabeculectomy. We have also tried to relate any changes found to changes in visual acuity. We only included patients with $6 / 12$ vision or better in order to enhance the assessment of subtle refractive changes. In addition, patients did not receive any cycloplegic drops post-operatively.

On pre-operative assessment the following details were recorded: uncorrected and corrected visual acuity; objective and subjective refraction performed by the same author (I.A.C.); axial length; keratometry; anterior chamber depth and corneal thickness; intraocular pressure using applanation tonometry; and fundal colour photography performed through a dilated pupil. Finally an oral fluorogram was performed using fluorescein capsules, at a concentration of $25 \mathrm{mg} / \mathrm{kg}$ body weight, with photography at 40 and 60 minutes. 4

Cairns-type trabeculectomy was performed in all cases. A fornix-based conjunctival flap was lifted and a partial tenonectomy performed. A $5 \times 3 \mathrm{~mm}$ partial-thickness rectangular scleral flap was dissected and a $4 \times 2 \mathrm{~mm}$ tra- 
Table I. Subjective refraction shown in dioptres as mean (standard deviation), and statistical significance

\begin{tabular}{lllll}
\hline & Sphere & $\begin{array}{l}\text { Initial with-the-rule } \\
\text { cylinder }\end{array}$ & $\begin{array}{l}\text { Initial against-the-rule } \\
\text { cylinder }\end{array}$ & $\begin{array}{l}\text { Spherical } \\
\text { equivalent }\end{array}$ \\
\hline Pre-operative & $+1.02(1.3)$ & $0.69(0.5)$ & $0.70(0.4)$ & $+0.67(1.3)$ \\
Week 1 & $-0.43(2.1) p<0.01$ & $1.81(1.0) \mathrm{NS}$ & $0.84(0.5) \mathrm{NS}$ & $-0.98(2.1) p<0.01$ \\
Week 3 & $+0.78(1.4) \mathrm{NS}$ & $1.06(0.5) \mathrm{NS}$ & $0.86(0.5) \mathrm{NS}$ & $+0.32(1.4) \mathrm{NS}$ \\
Week 8 & $+0.86(1.4) \mathrm{NS}$ & $1.12(0.6) \mathrm{NS}$ & $0.98(0.3) \mathrm{NS}$ & $+0.37(1.4) \mathrm{NS}$ \\
10 months & $+1.08(1.2) \mathrm{NS}$ & $1.25(0.5) \mathrm{NS}$ & $0.75(0.2) \mathrm{NS}$ & $+0.64(1.2) \mathrm{NS}$ \\
\hline
\end{tabular}

NS, not significant.

becular section removed anterior to the scleral spur. A peripheral iridectomy was performed. The scleral flap was sutured at the corners with two $10 / 0$ nylon sutures and a further two 10/0 nylon sutures were used to close the conjunctiva. Topical antibiotics were applied at the end of the procedure. Post-operatively the patients received a topical steroid four times a day for 4 weeks.

Full examination and all measurements, including photography and oral fluorograms, were repeated at 1, 3 and 8 weeks post-operatively. Topical tropicamide and phenylephrine were administered to ensure adequate mydriasis for photographic purposes only. A record of any interposing complications was made. including wound leak, choroidal detachments, or hyphaema. At long-term follow-up all measurements except an oral fluorogram were repeated.

\section{RESULTS}

Sixteen eyes of 16 patients were included in the study. Post-operative measurements were statistically compared with pre-operative measurements by paired $t$-test, and changes were analysed by simple, multiple and stepwise

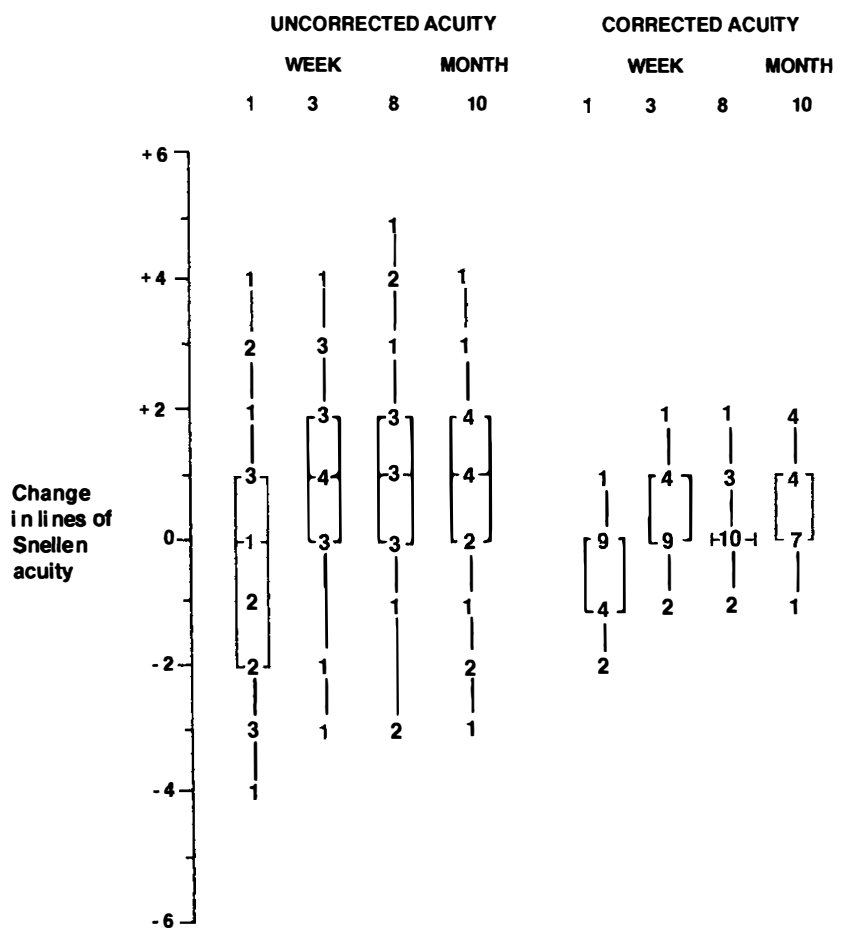

Fig. 1. Statistical comparison of the difference (in lines of Snellen) between pre-operative visual acuity and visual acuity at weeks 1,3 and 8 and at 10 months following trabeculectomy. regressions. Eight patients were male and eight female, with a mean age of 64.9 years. There were 8 right eyes and 8 left eyes in the study. The median axial length was 23.29 $\mathrm{mm}$, with a mean of $23.54 \mathrm{~mm}$. Fifteen patients had local anaesthetic and one general anaesthetic. The mean duration of glucoma from diagnosis to surgery was 3.15 years, and patients were on a mean of 1.8 topical medications per eye. Five eyes had previously undergone laser trabeculoplasty. Mean follow-up was 10.1 months (range 6-13). Mean intraocular pressure fell from $23.9 \mathrm{mmHg}$ (median $22.0 \mathrm{mmHg}$ ) pre-operatively to $14.1 \mathrm{mmHg}$ (median 14.0 $\mathrm{mmHg}$ ) at 10 months post-operatively.

Pre-operatively 13 eyes were hypermetropic and 3 eyes myopic. Mean pre-operative refractive sphere was +1.02 dioptres with a mean cylinder of -0.69 dioptres at $100^{\circ}$. The refractive sphere was significantly $(p<0.01)$ more myopic at week 1 with a mean of -0.43 dioptres, but was not significantly different from week 3 onwards. The spherical equivalent was also statistically more myopic at week $1(p<0.01)$. There was no significant change postoperatively in the axis or power of the refractive cylinder. If the subjective refractive cylinders are separated into those that were initially with-the-rule and those that were against-the-rule, again there was no statistically significant difference in power at any stage compared with preoperative values (Table 1).

The corrected visual acuity pre-operatively was $6 / 12$ or better in all cases and this was maintained through to final follow-up in all eyes except one, which had an acuity of $6 / 18$ due to posterior subcapsular cataract. Fifteen eyes (94\%) showed an alteration in uncorrected visual acuity, at week 1 , and $43 \%$ of eyes showed a change in corrected acuity. At weeks 3 and 8 only 2 eyes (12\%) had a reduction of one line of corrected Snellen acuity, and at final follow-up only one eye had an acuity that was less than its pre-operative level. Fig. 1 is a box-plot showing the changes in lines of Snellen acuity for each eye (corrected and uncorrected) compared with pre-operative levels over the follow-up period. Each figure represents the number of eyes that attained the given improvement or deterioration in lines of Snellen acuity at each follow-up visit.

The effect of the refractive changes on visual acuity is not apparent when the group is considered as a whole, as myopes and hypermetropes behave differently. We calculated the 'nett shift towards emmetropia' for the changes in spherical equivalent and refractive sphere from the preoperative state to week 1 , from week 1 to week 3 , from week 3 to week 8 , and from week 8 to final follow-up. This was done by taking the difference in the two refractive 


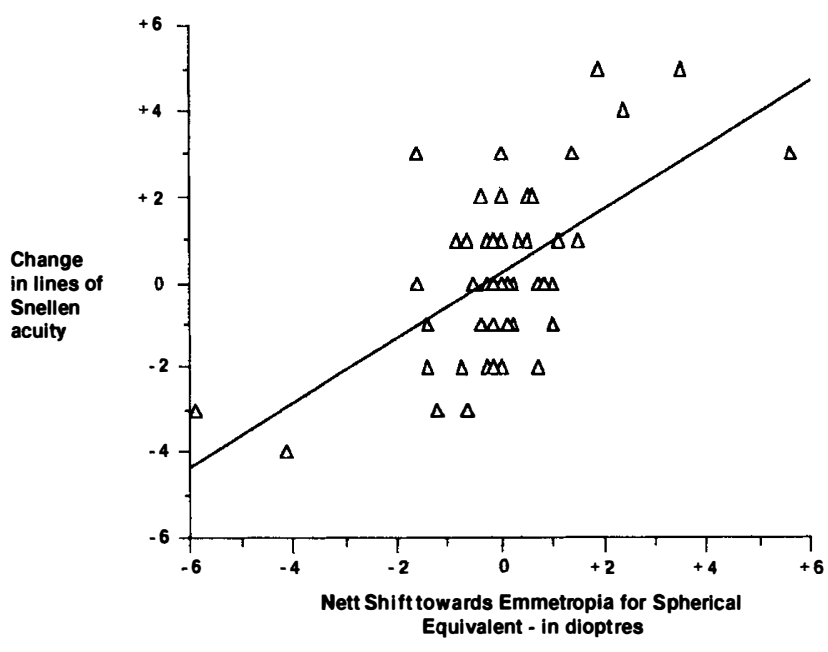

Fig. 2. Scatter plot showing the changes in uncorrected visual acuity (in lines of Snellen) in relation to changes in spherical equivalent with respect to emmetropia.

values and assigning a positive value if the difference in refraction was towards emmetropia and a negative value if it was away from emmetropia. This 'nett shift towards emmetropia' for the changes in spherical equivalent can be plotted against the changes in uncorrected visual acuity, as is shown in Fig. 2. The correlation coefficient $(R)$ for this plot is 0.60 . A similar plot of the changes in refractive sphere has a correlation coefficient of 0.62 . The subjective changes in vision can therefore be shown to be related to the changes in refractive sphere and spherical equivalent that occur in the post-operative period.

The vertical corneal radius of curvature (axis ranging from $46^{\circ}$ to $125^{\circ}$ ) was significantly smaller at the first three post-operative visits. Mean pre-operative vertical radius of curvature was $7.69 \mathrm{~mm}$ compared with $7.56 \mathrm{~mm}$ at week $1(p<0.02), 7.60 \mathrm{~mm}$ at week $3(p<0.05)$ and 7.58 $\mathrm{mm}$ at week $8(p<0.01)$. On final follow-up the mean vertical corneal radius of curvature was $7.71 \mathrm{~mm}$ and was not significantly different from pre-operative levels. The horizontal corneal radius of curvature was significantly increased at weeks 1 and $3(p<0.05)$, but not significantly different from week 8 onwards (Fig. 3). There was no statistical difference in the axis of the vertical corneal cur-

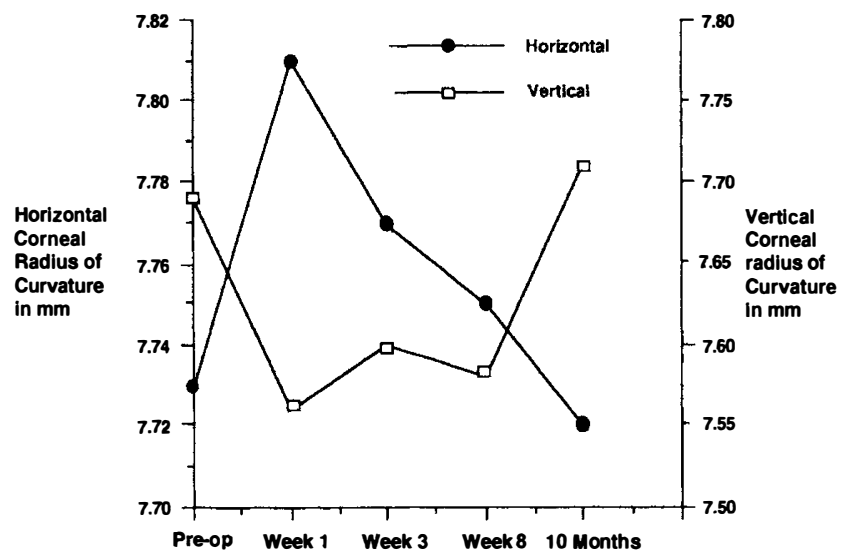

Fig. 3. Changes in vertical and horizontal corneal radii of curvature following trabeculectomy. vature at any of the post-operative assessments (Table II). The concept of polar values calculated from keratometer readings has been described by Naeser. ${ }^{5}$ The polar value calculates the balance between the with-the-rule and against-the-rule components for any given nett astigmatism, and enables corneal astigmatism to be expressed as a single figure. An increase in polar value denotes a withthe-rule change. Polar values showed a with-the-rule change at weeks 1,3 and 8 when compared with preoperative values, being statistically different at weeks 1 and 8; but this change had resolved by final follow-up assessment (Table II). These changes are consistent with a shortening of the vertical corneal radius of curvature.

Mean pre-operative anterior chamber depth was 2.81 $\mathrm{mm}$, and this became significantly $(p<0.001)$ shallower at week 1 with a mean of $1.74 \mathrm{~mm}$. At week $1,94 \%$ of eyes had shallower anterior chambers compared with pre-operative depths. In $80 \%$ of these eyes the shallowing was $\geqslant$ $0.5 \mathrm{~mm}$, and in $53 \%$ the shallowing was $\geqslant 50 \%$ of the preoperative depth. At weeks 3 and 8 and on final follow-up, $56 \%$ of eyes still had anterior chambers shallower than the pre-operative depth, but only one of these eyes $(6 \%)$ had a difference greater than $0.5 \mathrm{~mm}$, which occurred at week 3 . The mean anterior chamber depths at weeks 3 and 8 were $2.74 \mathrm{~mm}$ and $2.78 \mathrm{~mm}$ respectively and $2.79 \mathrm{~mm}$ on final follow-up (Table III). Anterior chamber depth changes can be shown to correlate with changes in the refractive sphere and spherical equivalent. Fig. 4 shows the changes in anterior chamber depth (in $\mathrm{mm}$ ) plotted against the corresponding change in refractive sphere. Data shown are for the changes that occurred from pre-operative values to week 1 , week 1 to week 3 , and week 3 to week 8 . The anterior chamber may have shallowed (a negative value) and subsequently deepened (a positive value); similarly the change in refractive sphere may have been myopic (a negative value) or hypermetropic (a positive value). This plot has a correlation coefficient $(R)$ of 0.79 . A similar plot for changes in spherical equivalent has a correlation coefficient of 0.83 . Multiple and stepwise regression analysis showed that the change in anterior chamber depth was the only parameter, of those measured, that correlated with the changes in refractive sphere at week $1(R=0.77)$, week $3(R=0.70)$ and week $8(R=0.48)$. A $1 \mathrm{~mm}$ change in anterior chamber depth results in approximately a 2 dioptre change in refractive sphere or spherical equivalent. No changes were noted in the corneal thickness at any time during the study (Table III).

Only 11 patients consented to oral fluorograms. The results were independently assessed by a retinal specialist. No pre-operative fluorograms showed any evidence of macular oedema. Ninety per cent of the post-operative fluorograms performed were of good enough quality to assess for macular oedema. A supero-temporal branch vein occlusion was present pre-operatively in one patient, and this case showed some macular leakage at week 8 . This eye was not included in the fluorogram assessment statistics for macular oedema as it had a predisposing condition. This eye was, however, included in the rest of the 
Table II. Central corneal radius of curvature (in $\mathrm{mm}$ ) and polar values shown as mean (standard deviation), and statistical significance

\begin{tabular}{|c|c|c|c|c|}
\hline & Horizontal radius & Vertical radius & Vertical axis & Polar values \\
\hline Pre-operative & $7.73(0.25)$ & $7.69(0.37)$ & $79.7(23.8)$ & $0.31(1.06)$ \\
\hline Week 1 & $7.81(0.20) p<0.05$ & $7.56(0.33) p<0.02$ & $88.3(20.6) \mathrm{NS}$ & 1.30 (1.41) $p<0.01$ \\
\hline Week 8 & $7.75(0.23) \mathrm{NS}$ & $7.58(0.33) p<0.01$ & $89.8(25.0) \mathrm{NS}$ & $0.68(0.96) p<0.05$ \\
\hline 10 months & $7.72(0.22) \mathrm{NS}$ & $7.71(0.31) \mathrm{NS}$ & $92.6(25.2) \mathrm{NS}$ & $0.25(0.85) \mathrm{NS}$ \\
\hline
\end{tabular}

NS, not significant.

study as pre-operatively there was no evidence of macular oedema and the eye had an acuity of $6 / 5$. No other fluorograms showed any evidence of macular oedema over the follow-up period.

At week 1, 3 eyes had evidence of residual hyphaema, 10 eyes had peripheral choroidal detachments and 3 eyes had evidence of a wound leak on Siedel test. The eyes with wound leaks did not have anterior chambers that were shallow enough to require surgical correction. By week 3 no eyes showed evidence of hyphaema, choroidal detachment or wound leak.

\section{DISCUSSION}

Subjectively, patients may report an improvement or a deterioration in vision following surgery, and this is dependent upon their initial refractive state. Most eyes showed a myopic shift in refraction at week 1 that had almost returned to pre-operative levels by week 3 . The effect of this myopic shift on vision depends on the preoperative refractive state. Most eyes that were myopic preoperatively became more myopic with a corresponding fall in uncorrected visual acuity. The degree of pre-operative hypermetropia affected the uncorrected visual acuity changes in the hypermetropes. Hypermetropes in which the myopic shift resulted in a refraction closer to emmetropia, tended to show an improvement in uncorrected acuity. Hypermetropes whose myopic shift resulted in a refraction further away from emmetropia showed a fall in uncorrected visual acuity. At week 3 , when the myopic shift had returned towards pre-operative levels, changes in acuity again depended upon changes in the refraction with respect to emmetropia. We have shown that the subjective changes in vision are principally related to the changes in the refractive sphere and spherical equivalent that occur in the post-operative period.

Previous studies ${ }^{6-9}$ have shown that the anterior chamber tends to shallow in the post-operative period following trabeculectomy, and that maximal shallowing occurs by the fifth day. Most anterior chambers tend to re-form spon-

Table III. Anterior chamber depth and corneal thickness (in $\mathrm{mm}$ ) shown as mean (standard deviation), and statistical significance

\begin{tabular}{lll}
\hline & $\begin{array}{c}\text { Anterior chamber } \\
\text { depth }\end{array}$ & \multicolumn{1}{c}{$\begin{array}{c}\text { Corneal } \\
\text { thickness }\end{array}$} \\
\hline Pre-operative & $2.81(0.21)$ & $0.58(0.05)$ \\
Week 1 & $1.74(0.82) p<0.001$ & $0.58(0.06) \mathrm{NS}$ \\
Week 3 & $2.74(0.30) \mathrm{NS}$ & $0.58(0.04) \mathrm{NS}$ \\
Week 8 & $2.78(0.27) \mathrm{NS}$ & $0.59(0.03) \mathrm{NS}$ \\
10 months & $2.79(0.25) \mathrm{NS}$ & $0.58(0.04) \mathrm{NS}$ \\
\hline
\end{tabular}

NS, not significant. tanenously by 2 weeks after surgery. Raitta ${ }^{10}$ has reported a 'trend' towards less anterior chamber shallowing with the use of sodium hyaluronate. However, no actual anterior chamber depth measurements are given in this study. The anterior chamber depth changes have not previously been correlated with early changes in refraction following trabeculectomy. The anterior chamber depth changes in our study are similar to those of other studies, with a significant shallowing being apparent at week 1 and resolving by week 3 . When the anterior chamber shallows the ciliary body may move forward, which in combination with any ciliary spasm will release the tension in the zonules and may allow the lens to accommodate with a resultant change in the position and thickness of the lens. In this study we did not measure any changes in lens thickness. The mean amplitude of accommodation in the age group of this study is approximately 1 dioptre. ${ }^{11}$ Some of this accommodation may be contributing to the overall myopic shift in refraction. The anterior chamber depth changes have been shown to be the principal cause for refractive (and subsequent visual) changes that occur following trabeculectomy. The visual acuity starts to return to normal by the third post-operative week, provided that the anterior chamber has also returned to pre-operative depths.

The change in vertical corneal curvature is consistent with the with-the-rule change in polar values, and both are in keeping with a with-the-rule change in corneal astig-

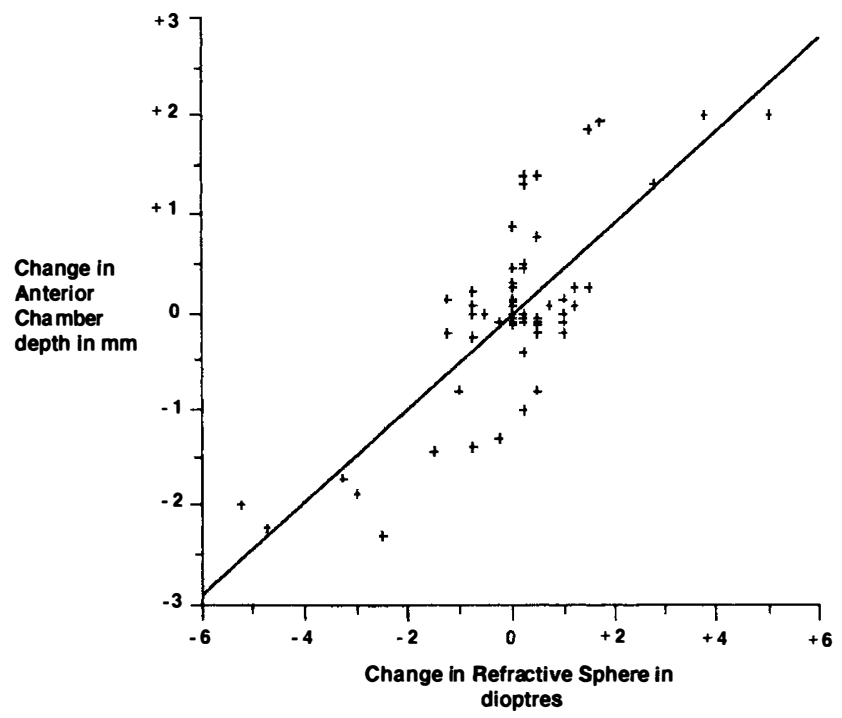

Fig. 4. Scatter plot showing the changes in refractive sphere in relation to changes in anterior chamber depth following trabeculectomy. 
matism following trabeculectomy as shown on corneal topography. ${ }^{12,13}$ The reason for this change following drainage surgery is not known. Hugkulstone ${ }^{14}$ looked at corneal curvature following trabeculectomy in 10 patients and found similar changes in the vertical corneal radius. He postulated that these changes may be due to the posterior site of the surgically induced wound gape in trabeculectomy, and also the support offered by the overlying scleral flap. However, we feel that this wound gape should lead to an increase in the vertical corneal radius of curvature. It may be in trabeculectomy, that when a $2 \times 4 \mathrm{~mm}$ piece of tissue is removed from under the scleral flap and the flap then sutured back, that the flap approximates the corneal edge of the trabeculectomy opening to the scleral edge and also allows the unsupported corneal edge of the trabeculectomy opening to sink slightly. Both of these mechanisms would have the effect of shortening the vertical corneal radius of curvature. On final follow-up the vertical corneal curvature had returned to pre-operative levels. From 2 months onwards the tension in the scleral sutures may start to weaken and allow the free corneal edge of the sclerostomy opening to resume its pre-operative position.

To our knowledge, the incidence of macular oedema post-trabeculectomy has not been studied. We used oral fluorograms with late photography to look for leakage at the macula. In $90 \%$ of the fluorograms performed the quality was good enough to allow assessment for macular oedema. Although the numbers are small in this study, we found no evidence of post-operative macular oedema in the fluorograms that were included in the study, and therefore do not consider macular oedema to be a significant factor contributing to the early visual changes following trabeculectomy. No eyes in this study had hypotony (which can cause macular oedema) in the post-operative period. Clinical examination of the macula also failed to reveal any abnormalities. Surface-wrinkling maculopathy has been reported ${ }^{15}$ to occur following trabeculectomy.

We found no changes in corneal thickness at any time in the follow-up period. One previous report ${ }^{16}$ has shown that a small thickening (approximately $0.01 \mathrm{~mm}$ ) of central corneal thickness occurs at days 1 and 4 post-trabeculectomy. We measured corneal thickness at week 1 and would have missed this change.

We have shown that a large percentage of patients have a subjective change in vision following trabeculectomy. In the majority of cases this resolves within 8 weeks of surgery, at which stage no patients showed a deterioration in best corrected visual acuity of more than one line Snellen acuity. The principal cause of these changes in vision is the shallowing of the anterior chamber that occurs in the first week following surgery. This results in a myopic shift in refraction that starts to return to normal within 3 weeks. A small change in the vertical corneal radius of curvature appears to persist for more than 2 months post-operatively, and this is associated with a with-the-rule change in corneal astigmatism. Both these changes appear to resolve over the next few months. No significant changes were found in any of the other factors assessed. Patients should be warned of these changes pre-operatively as many will have normal vision prior to surgery and may be distressed by the significant myopic changes that can occur.

We would like to thank Mr. C. Mody (ophthalmic photographer) for performing the oral fluorograms and fundal photography, Mr. J. F. Talbot (consultant ophthalmologist) for his assessment of the angiograms, and Sister McCann and her staff for their assistance in out-patients.

Key words: Anterior chamber depth, Keratometry, Refraction, Trabeculectomy, Visual acuity.

\section{REFERENCES}

1. Cairns JE: Trabeculectomy: preliminary report of a new method. Am J Ophthalmol 1968, 66, 673-9.

2. Watson PG: Trabeculectomy: a modified ab externo technique. Ann Ophthalmol 1970, 2: 199-205.

3. Watson PG, Grierson I: The place of trabeculectomy in the treatment of glaucoma. Ophthalmology 1981, 88: 175-96.

4. Watson AP, Rosen ES: Oral fluorescein angiography: reassessment of its relative safety and evaluation of optimum conditions with use of capsules. Br J Ophthalmol 1990, 74: $458-61$.

5. Naeser K. Conversion of keratometer readings to polar values. J Cataract Refract Surg 1990, 16: 741-5.

6. Kao SF, Litcher PR, Musch DC: Anterior chamber depth following filtration surgery. Ophthalmic Surg 1989 20: 332-6.

7. Mackool RJ, Buxton JN: Anterior chamber depth after intrascleral filtering surgery. Ophthalmic Surg 1977, 8: 40-4.

8. Stewart WC, Shields MB: Management of anterior chamber depth after trabeculectomy. Am J Ophthalmol 1988, 106: $41-4$.

9. Goins K, Smith T, Kinker R, Lewis J: Axial anterior chamber depth after trabeculectomy. Ophthalmologica 1990, 200: $177-80$.

10. Raitta C, Vesti E: The effect of sodium hyaluronate on the outcome of trabeculectomy. Ophthalmic Surg 1991, 22: 145-9.

11. Duke-Elder S: Practice of Refraction, 7th ed. London: Churchill, 1963: 141.

12. Rosen WJ, Mannis MJ, Brandt JD: The effect of trabeculectomy on corneal topography. Invest Ophthalmol Vis Sci 1992, 33/4 (Suppl): 1515.

13. Dietze PJ, Koch DD, Gross RL: Corneal topography after trabeculectomy. Invest Ophthalmol Vis Sci 1992 33/4 (Suppl): 2343.

14. Hugkulstone CE: Changes in keratometry following trabeculectomy. Br J Ophthalmol 1991, 75: 217-18.

15. Detry-Morel M, Kitell B, Lemagne JM: Surface-wrinkling maculopathy as a potential complication of trabeculectomy: a case report. Ophthalmic Surg 1991, 22: 38-40.

16. Bramsen T: A double-blind study on the influence of tranexamic acid on the intraocular pressure and central corneal thickness after trabeculectomy for glaucoma simplex. Acta Ophthalmol (Copenh) 1978, 56: 998-1005. 